\title{
Keilschrift, Kant und Kaufverträge
}

\author{
Von der Philologie zur Medienkompetenz und zurück
}

Korrespondenzadresse

Prof. Dr. Dr. Manfred Spitzer

Universität Ulm

Abteilung für Psychiatrie

Leimgrubenweg 12-14

$87054 \mathrm{Ulm}$
Bibliografie

DOI https://doi.org/10.1055/a-1033-9748

Nervenheilkunde 2020; 39: 198-205

(c) Georg Thieme Verlag KG Stuttgart · New York

ISSN 0722-1541
„Die Erfindung der Schrift vor mehr als 5 Jahrtausenden veränderte die Gesellschaften zwischen Tigris und Nil grundlegend. Die Einführung der Hieroglyphenschrift in Ägypten und der Keilschrift in Mesopotamien war eine Innovation mit weitreichenden Folgen für das Zusammenleben der Menschen, für Kommunikation und Kontrolle, Planung und Organisation, Erinnerungskultur und Traditionsbildung, Arbeitswelt und soziale Strukturen, künstlerische Ausdrucksformen und nicht zuletzt für die Speicherung, Verarbeitung und Übermittlung von Wissensbeständen.“

Ersetzt man in diesem Zitat aus dem sehr lesenswerten Buch „Erste Philologien“ von Eva Cancik-Kirschbaum und Jochem Kahl [4] (॰ Abb. 1) über die Anfänge der Philologie das Wort „Schrift“ durch „Computer“, kann man alles so stehen lassen. Dies bedeutet auch, dass wir uns mit den Prozessen des Verstehens, der Speicherung und der Weitergabe von Wissen nicht erst seit einigen Jahrzehnten, sondern seit mehr als 4 Jahrtausenden beschäftigen. Man nennt dieses Sachgebiet seit etwa 2 Jahrhunderten Philologie.

Zur Geschichte sei in systematischer Hinsicht kurz erwähnt, dass das Fehlen eines Begriffs in einer alten Sprache keineswegs bedeuten muss, dass über die mit ihm gemeinten Sachverhalte nicht nachgedacht worden wäre. „Beispielsweise war Religion in Ägypten und Mesopotamien allgegenwärtig, allerdings wird man in den modernen Wörterbüchern vergeblich nach einem Begriff dafür suchen“, verdeutlichen Cancik-Kirschbaum und Kahl [4] dies an der Religion und übertragen den Gedanken auf die Philologie. Das Wort wurde auch in der griechisch-römischen Antike nicht in der heute üblichen Bedeutung verwendet, die sich erst im vorletzten Jahrhundert herauskristallisierte. Noch Kant verstand darunter eher so etwas wie „Universalgelehrtentum“, Hegel sprach von einem unwissenschaftlichen Disziplinen-Aggregat, und für Wilhelm von Humboldt war Philologie all das, was man heute unter „Geisteswissenschaft“ und im angloamerikanischen Sprachraum unter „Humanities“ versteht [7].

Die Philologie als Wissenschaft vom Verstehen, die sich sowohl um Grammatik, Psychologie, Geschichte und Kritik eines Textes kümmert und diese Aspekte des Verstehens reflektiert, gibt es seit etwa 200 Jahren, wobei verschiedene Autoren und mittlerweile Wissenschaftszweige unterschiedliche Schwerpunkte setzten. Studiert man heute lateinische, griechische oder orientalistische Philologie (z. B. die Assyriologie), dann paukt man zunächst die Spra- chen (und Schriften), d. h. deren Wörter und deren Bedeutungen (Semantik) und die Struktur der Sprachen (Grammatik). Wussten Sie, dass man Keilschrift nur dann wirklich lesen kann, wenn man auch etwa ein Dutzend Sprachen beherrscht, die in Keilschrift aufgeschrieben wurden? Studiert man Psychologie, geht es um das Nacherleben und Nachvollziehen von Gefühlen und Gedanken (einschließlich der Frage, inwieweit hier Allgemeingültigkeit bestehen kann). Und studiert man Religion oder Geschichte, geht es eher um das Verstehen von Inhalten im Sinne einer Rekonstruktion der hinter vielfältigen Überlieferungen herauszufindenden Wahrheit (und aller Probleme, die damit verbunden sind).

\section{Schreiben in Schulen}

Das Schreiben wurde bereits vor knapp 4000 Jahren in Schulen gelehrt, wo 3 bis höchstens 12 Schüler gemeinsam unterrichtet wurden, wie man aus Schriften hierzu und Funden von Tontafeln weiß, bei denen es sich um das handelte, was man heute „Übungshefte“ nennt. Im von der Bayrischen Akademie der Wissenschaften online zur Verfügung stehenden Reallexikon der Assyriologie und vorderasiatischen Archäologie findet man unter dem Stichwort „Schule“ das Folgende: „Mit der Erfindung der Keilschrift ergab sich die Notwendigkeit, diese zu erlernen. Die kundigen Schreiber mussten ihre Fähigkeiten an die nächste Generation weitergeben. Dazu entwickelten sie im Laufe der Zeit unterschiedliches Unterrichtsmaterial, das immer wieder an die Erfordernisse angepasst wurde. [...] Übungen von Elementarschülern sind durch unsichere, große Schrift zu erkennen. [...] der Unterricht begann mit dem Einüben der Elemente der Schriftzeichen. Danach wurden diese zu richtigen Schriftzeichen zusammengesetzt. [...] Viel Übung bedurften die komplizierten Zahlzeichen der Maßeinheiten und die verschiedenen Rechenarten. Buchhaltung und Erstellen von Abrechnungen erlernten die Schüler anhand von Texten mit dem Aufbau von normalen Verwaltungstexten“ [19]. „Die Kinder besuchten wohl ab dem Alter von 5 bis 7 Jahren die Schule“.

Vor 4 Jahrtausenden mussten sich die Schüler also auch schon mit aus ihrer Sicht „langweiligem und uraltem Kram“ aus der Verwaltung und den Jahrhunderten davor befassen. In ganz Assyrien 


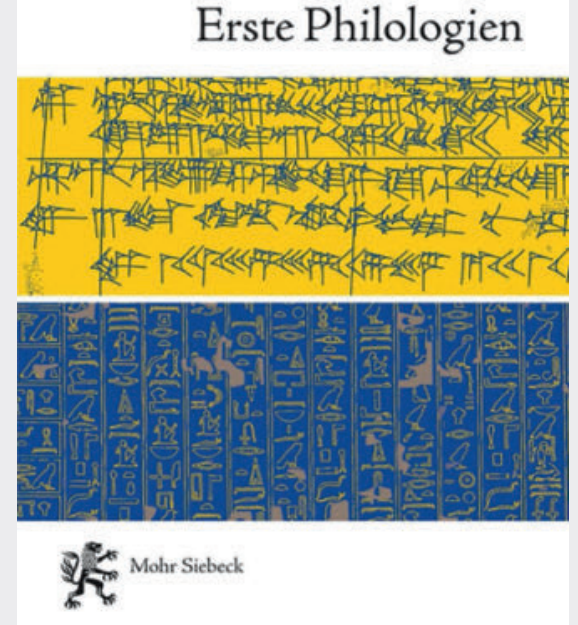

- Abb. 1 Cover des Buchs „Erste Philologien“ von Eva CancikKirschbaum und Jochem Kahl.

war das Curriculum sehr ähnlich ${ }^{1}$ (wie man anhand der vielen gefunden Tontafeln zeigen kann) und übrigens von Beginn an 2-sprachig: „Der allergrößte Teil des Unterrichtsstoffs während der Elementarausbildung war Sumerisch, obwohl die Schüler - soweit bekannt - Akkadisch als Muttersprache hatten“ [19]. Schon damals war die Schule also für Kinder eine anstrengende große geistige Herausforderung. Sie lernten auch, in ihrer Muttersprache zu schreiben. Die Haptik des Schreibens auf Tontafeln war komplizierter als Papier, Bleistift und Radiergummi, und die Inhalte - Schreiben, Lesen, Rechnen, Büroarbeit - abstrakt. Nur wenige gingen zur Schule und noch weniger brachten es nach dem Elementarunterricht (der reichte für Beamte) zum Studium dessen, was man heute „literarische Texte“ (einschließlich Schuldialoge und Streitgespräche) nennen würde. Die Schule fand übrigens an 24 Tagen im Monat statt, „an 3 Tagen hatten sie frei und an 3 weiteren Tagen fanden Feste statt“" [19] Richtig zeitgenössisch mutet folgende Passage an: „In einigen der als Schule identifizierten Gebäuden fand man aus gebrannten Ziegeln gebaute Behälter, in denen alte Schulübungen lagen. Der Ton sollte recycelt und zu neuen Tafeln geformt werden [19]. Auch die heute viel beklagte Abhängigkeit des erreichten Bildungsgrads von der sozialen Schicht gab es längst: „Literarische Bildung scheint wenigen vorbehalten gewesen zu sein, vornehmlich Söhnen von Vätern in Spitzenpositionen in Tempel, Palast oder Verwaltung“ [19].

1 „Von einer Stadt zur anderen sind die Schultexte in Aufbau und Inhalt so ähnlich, dass man beinahe von einem einheitlichen Programm sprechen kann“ steht dazu in der gleichen Quelle (S. 306) geschrieben, mit dem zusätzlichen Hinweis, dass das System sogar im Nachbarland Babylon sehr ähnlich war.
Der Alltag von Schülern war damit vor 4000 Jahren dem von heute keineswegs unähnlich, nur in vielfacher Hinsicht zielgerichteter und wahrscheinlich effektiver. „Auf jeden Fall gehörten Abschreiben, schriftliches und mündliches Wiederholen und Auswendiglernen zu den wichtigsten Unterrichtsmethoden. Die Schüler mussten auch nach Diktat schreiben, wie man u. a. an typischen Diktatfehlern erkennt. [...] Streitgespräche sollten die Ausdrucksfähigkeit fördern“ [19].

Diese kurze Darstellung dessen, was „Schule“ vor 4 Jahrtausenden bei den Assyrern bereits war, macht deutlich, worum es damals beim Schreiben, übrigens parallel in Ägypten mittels der Hieroglyphen, ging: Medienkompetenz, wie wir heute sagen würden. Zunächst wurden weder Gedichte noch Romane geschrieben, sondern Listen „zur Überwachung und Protokollierung des gespeicherten Getreides [4]. Rechnungen, Quittungen, Kauf- und Pachtverträge, Geschäftsbriefe, Testamente, Gerichtsprotokolle und der ein oder andere Liebesbrief. Was gehört wem? Was war los? Was wird wofür gebraucht? Wieviel Essen ist (noch) da? - Das Schreiben diente der ganz konkreten Lebensbewältigung und Lebenspraxis innerhalb von Städten, die mit ihren mehreren tausend Einwohnern ohne Schrift schwer existieren konnten. Bald kamen Verhaltenskodizes, religiöse Texte und Anweisungen (Gesetze) hinzu, die ebenfalls ganz praktisch den Austausch von Waren und Dienstleistungen, Gebräuche und Rituale zwischen einer immer größer werdenden Zahl von Menschen regelten.

Schreiben und lesen konnten nur wenige, weswegen sie sehr geschätzt waren und in den jeweiligen Staaten hohes Ansehen hatten. Man nannte sie „Schreiber“, was zugleich jedoch etwa die Bedeutung von „Beamter“ hatte. ${ }^{2}$ Und umgekehrt gilt: Hätte es nicht einen wirtschaftlichen Aufschwung mit zunehmender Produktivität der Landwirtschaft gegeben, die mehr Menschen ernähren konnte und deswegen größere Menschenansammlungen (Städte) überhaupt erst ermöglichte, wäre die Schrift gar nicht erfunden worden. Kleine Gruppen von Menschen brauchen sie nicht zum Funktionieren - Staaten schon. Die neue Art der Vermittlung von Wissen, nicht mehr durch unmittelbares Erzählen, sondern vermittelt durch das Schreiben auf und Lesen von Tontafeln, machte Schule erforderlich, die unseren heutigen Schulen sehr ähnlich war. Der Grund dafür ist einfach: So funktioniert Lernen beim Menschen nun einmal am besten: In jungen Jahren, unter Anleitung eines Lehrers, mit viel Übung und Wiederholung, sehr viel Praxis, und mit anwendungsorientierten Aufgaben, die dem Alltag entnommen sind.

Sobald es die Schrift gab und damit eine anfassbare und vom einzelnen Menschen unabhängige Form der längerfristigen Speicherung von Information, machten sich die Menschen aber nicht nur Gedanken zur praktischen Anwendung von Texten, sondern auch darüber, was ein Text ist, wie man ihn strukturiert, den Inhalt zugänglich macht und ihn langfristig erhält. So entstanden mit den Texten nahezu gleichzeitig auch die ersten Philologien, d. h. Gedanken zu deren Struktur, Funktion und Speicherung. Hinzu kommt: Wer so gut gebildet war, wie man das in den damaligen Schulen erlebt hatte, der machte sich Gedanken über „Bildung“. Und dies geschah über Jahrtausende der Schriftverwendung mit kontinuierlich zunehmender Professionalisierung im Umgang mit Texten. Große

2 Auch wir sprechen ja von „Schreibstube“, wenn wir ein Amtszimme meinen. 
Bibliotheken, wie sie nicht nur im ägyptischen Alexandria und im assyrischen Ninive, sondern an Dutzenden von Standorten in beiden Ländern entstanden [4] beinhalteten kodierte Wissensbestände, die es zu verstehen und zugänglich zu machen galt.

\section{Der hermeneutische Zirkel}

Über gut 4 Jahrtausende währende Gedanken dazu, wie man mit Texten umgeht und sie versteht, sind nicht Nichts. Fassen wir daher kurz einige grundlegende Erkenntnisse der Menschheit zu Texten zusammen:

- Ein Text ist nur dann ein Text, wenn man ihn versteht. Das hört sich trivial an, ist es aber nicht: Die Hieroglyphen waren so lange Ornamentik, also „nettes Verzierungsbeiwerk“ wie man sie nicht verstanden hatte. Erst ihre Entzifferung machte die Bildchen zu Zeichen, die für etwas stehen. Bis heute gibt es „Zeichen“, die man nicht entziffert hat und von denen daher niemand sagen kann, ob es nicht vielleicht doch nur Ornamente sind.

- Um einen Text zu verstehen, braucht man Vorwissen. Dieses bezieht sich zum einen auf das Wissen um die Bedeutung der Zeichen, das wiederum nicht in einzelnen „Bedeutungsschnipseln“ besteht, sondern in Zusammenhängen und Anwendungen: Was eine Gabel ist, weiß ich dann, wenn ich weiß, wie man sie beim Essen verwendet. Wenn eine Zivilisation auf die Erde käme, die sich durch Strom aus Steckdosen mit Energie versorgt, also keine chemische Energie (in Form von Nahrung) im Rahmen kulturell stark überformter Zeremonien (Mahlzeiten) zu sich nimmt, würde sie vielleicht Gabeln finden und darüber sinnieren, wozu sie gebraucht werden: Als Antennen für Signale? Als Kinderspielzeug im Sand? Als religiöses oder Kunstobjekt? Kurz: Solange unklar ist, was Stoffwechsel, was Nahrung und was eine Mahlzeit ist, wird sich die Bedeutung von dem Ding „Gabel“ nicht erschließen.

- Dieses Vorwissen besteht aus allgemeinem Weltwissen, zu dem Einzelheiten (Rom ist die Hauptstadt von Italien) und Allgemeinheiten $(2+2=4)$ gehören, die man ebenfalls anhand von Einzelheiten lernt (2 Äpfel und noch 2 Äpfel sind 4 Äpfel). Das Verallgemeinern ist dabei die Leistung eines jeden einzelnen Lernenden, und jeder ist beständig dabei, Erfahrungen zu verarbeiten, d. h. zueinander und mit dem, was man schon weiß, in Beziehung zu setzen, Widersprüche aufzudecken, durch Nachdenken oder das Suchen neuer Erfahrungen (d. h. Lernen) zu eliminieren, um sich so besser (d. h. erfolgreicher) in der Welt zurecht zu finden.

- Erst durch das Schreiben und Lesen wurde garantiert, dass nicht jede Generation wieder bei null anfangen muss, wenn z. B. durch Krieg oder Krankheit fast alle Wissenden hinweggerafft wurden. Wer schreiben und lesen kann, der kann sich selber wieder Informationen beschaffen und ist nicht auf andere, die etwas schon wissen, angewiesen: Wenn ich das Kochbuch meiner Großmutter habe, lesen kann und genügend Vorwissen habe (was bedeuten die Wörter „kochen“, „Teig“, „Speck“, „würfeln“?), erlaubt mir das auch ohne Anwesenheit meiner Oma nicht an Hunger zu versterben.

- Für den Umgang mit Texten ist Vorwissen unverzichtbar. Man kann es nicht aus den Texten gewinnen, sondern muss es schon haben, um die Texte zu verstehen. Hieraus ergibt sich ein scheinbar paradoxer Sachverhalt: Wer etwas Neues lernt, muss schon Vorwissen haben. Wenn man aber zum Lernen Vorwissen braucht und Vorwissen nur durch Lernen erworben werden kann, wie kommt das Ganze dann überhaupt in Gang?

Antworten auf diese Frage geben heute die Entwicklungspsychologie und die Gehirnforschung. Kinder sind ungemein lernfähig, verstehen sehr früh Gesten und Mimik, können ihre Aufmerksamkeit auf das richten, worauf ein Erwachsener schaut oder mit dem Finger deutet, und erwerben so durch mit anderen „geteilte Aufmerksamkeit“ die Namen und den Gebrauch von Dingen, Handlungen und Vorgängen. Sie haben Spaß am Verallgemeinern und suchen ständig neue Erfahrungen. Die Gehirnforschung hat verstanden, dass Gehirne sich durch ihren Gebrauch ändern und dadurch Spuren (Gedächtnisspuren) entstehen, d. h. Muster aus stärkeren und schwächeren Synapsen (Verbindungen zwischen Nervenzellen), welche die gemachten Erfahrungen intern repräsentieren. Durch Anwendung und Neukombination solcher Muster kann ich mit ihnen nicht nur die Vergangenheit und Gegenwart verstehen, sondern vor allem auch die Zukunft planen.

Die Antwort der Philologie auf diese Frage ist unter dem Namen „hermeneutischer Zirkel“ seit mehr als 150 Jahren bekannt: Man fängt irgendwo an, macht in einem neuen Sachgebiet erste unzusammenhängende Erfahrungen (z. B. „da bewegt sich was selbsttätig“), bemerkt Zusammenhänge, Ähnlichkeiten und Unterschiede (also Relationen zwischen den Dingen) und verwendet diese zur (ersten und vorsichtigen) Generalisierung und Strukturierung der Erfahrungen (z. B.: „es gibt so etwas auf dem Land, in der Luft und im Wasser“). Man verschafft sich also eine Übersicht, indem man Landtiere von Vögeln und Fischen unterscheidet, und damit kommt man lebenspraktisch schon ziemlich weit: Man kann das alles essen, muss sich fürchten, wenn es groß ist und vor allem große Zähne hat etc. Die Einteilung erlaubt auch Vorhersagen (was fliegt, legt Eier; auch die sind essbar), und wenn die manchmal nicht zutreffen (Fledermäuse fliegen, legen aber keine Eier; Schildkröten legen Eier, fliegen aber nicht) lernt man wieder etwas dazu. Man beachte: Nur wer vorher verallgemeinert, stolpert über falsche Vorhersagen, und nur deswegen wird gelernt, $d$. h. eigenes Wissen generiert. Dieses ist grundsätzlich immer vernetzt und anwendungsrelevant, denn nur durch das Herstellen von Beziehungen zwischen einzelnen Erlebnissen und deren Anwendung auf neue Erlebnisse werden diese zu Erfahrung. Das Auswendiglernen eines Telefonbuchs generiert daher kein Wissen. Wissen ist damit tiefer als einzelne Erlebnisse, denn äußerlich Ähnliches (Thunfisch und Delfin) kann völlig verschieden sein (Fisch versus Säugetier) und äußerlich sehr Unähnliches (Maus und Elefant) kann sehr ähnlich sein. Je mehr Vorwissen ich habe, desto tiefer kann mein Verständnis sein. Das ist die grundlegende Einsicht, die hinter dem Namen hermeneutischer Zirkel steckt. Man fängt irgendwo an, und meistens hat man schon angefangen, weil man die Kindheit und Jugend schon hinter sich hat. Geschieht es jedoch, dass ein Erwachsener plötzlich fremden Menschen, Sachen oder Schriftzeichen begegnet, dann wird er den hermeneutischen Zirkel hautnah erleben. Dies geschah im 
18. und 19. Jahrhundert sehr häufig, weswegen man sich auch in dieser Zeit so intensive Gedanken darüber machte. ${ }^{3}$

\section{Medienkompetenz?}

Diese Gedanken sind bis heute nicht falsch, aber zuweilen vollkommen vergessen. Betrachten wir ein Beispiel: Es scheinen sich alle am Digitalisierungsgeschehen Beteiligten einig darüber zu sein, dass die Menschen mehr Medienkompetenz haben sollten. Damit stellt sich die Frage: Was ist das? - Manche sprechen auch vom „Internetführerschein“ oder von „Informationskompetenz“. Fragt man dann genauer nach, so erhält man eine Reihe von Antworten: „Wir brauchen mehr Medienkompetenz, um Fake-News zu entlarven; um Suchmaschinen bedienen zu können und relevante Informationen zu finden (und von irrelevanten unterscheiden zu können); um an Informationen irgendwo in der Cloud heranzukommen und sie bewerten; um wahre von falschen Aussagen unterscheiden zu können.“ Daher sei „Medienkompetenz“ eine „unverzichtbare Kernkompetenz“ bzw. eine sehr wichtige „Kulturtechnik“ - wie etwa Lesen, Schreiben und Rechnen.

Das Ganze erscheint bei flüchtiger Betrachtung vernünftig und sinnvoll, hat jedoch einen entscheidenden Haken: Eine allgemeine Fähigkeit, Wahrheit von Falschheit zu unterscheiden, gibt es nicht, weil es eine solche Fähigkeit gar nicht geben kann. Ein jeder - Bauer, Bäcker oder Mathematiker - kennt sich irgendwo aus, und kann damit auch sofort wahre von falschen Aussagen über Getreideanbau, Brot oder die Kreiszahl unterscheiden. Eben weil - und nur weil - er über das entsprechende Hintergrundwissen verfügt. Um ein Urteil über die Wahrheit oder Falschheit einer Aussage über irgendetwas - völlig egal, worum es geht - zu fällen, braucht man ganz grundsätzlich Vorwissen über das Sachgebiet, in das diese Aussage eingebettet ist.

Eine Fähigkeit, die Wahrheit oder Unwahrheit von Informationsschnipseln jeglicher Herkunft diesen selbst - ohne jegliche Vorkenntnisse - sozusagen sofort anzusehen (und die nicht mit Intelligenz, Denkvermögen, Durchhaltevermögen oder Willenskraft identisch ist, denn diese Fähigkeiten gibt es ja schon, weswegen sie auch schon einen Namen haben), gibt es nicht. Man kann daher auch nicht Googeln lernen. Es ist vielmehr das Wissen in einem bestimmten Fachgebiet (Seinsbereich), welches einem das Verständ-

3 Nicht von ungefähr fällt in diese Zeit auch die Entschlüsselung der Hieroglyphen und der Keilschrift. Mein Beispiel mit den Lebewesen habe ich bewusst gewählt, um zu verdeutlichen, dass es bei der Hermeneutik um ganz allgemeines lebenspraktisches Verstehen geht. „Mit der Nase auf diese Kenntnisse draufgestoßen“ wird aber nicht der Fischer, sondern der Philologe, der vor Papyrus oder Tontafeln sitzt und zunächst nichts versteht. Irgendwo fängt man dann an: ein Kreis mit einem Punkt darin könnte „Auge“ bedeuten und ein Ochse meint vielleicht einen Ochsen und ein Vogel meint vielleicht einen Vogel. In der Tat waren Keilschrift und Hieroglyphen in ihrer Anfangszeit vor mehr als 5000 Jahren Bilderschriften (aus Piktogrammen, also Bildern von Dingen oder Funktionen: 2 Beine bedeuteten „Iaufen“). Ideogramme (Zeichen für etwas ohne jegliche bildliche Ähnlichkeit von Zeichen und bezeichnetem Gedanken oder Begriff) und Logogramme (Zeichen für Wörter) kamen hinzu. Im Laufe der Jahrtausende wurden sie zu Silbenschriften und gegen Ende ihres Gebrauchs gab es auch Hieroglyphenund Keilschrift-Alphabete (mit Zeichen, die für Laute stehen: Phonogramme). All dies musste man erst einmal erkennen! nis von Einzelheiten in diesem Fachgebiet erlaubt. Solches Wissen besteht nicht in einer strukturlosen Ansammlung von irgendwelchen Fakten (Schnipseln wie etwa die Antwort auf die Frage „Welcher hinterindische Nacktfrosch kann bei minus 4 Grad Celsius kopulieren?“), sondern ist grundsätzlich, wie in der Philologie schon lange bekannt, vernetzt und handlungsrelevant: Wir haben ein Gesamtbild, vor dessen Hintergrund wir aufgrund unseres allgemeinen Wissens und unserer Erkenntnisse über einen speziellen Fall (wie man dieses Brot backt, diese Brücke baut oder wie man diesen Menschen medizinisch versorgt) zum Handeln fähig sind.

Bereits im Jahr 2011 wurden von Psychologen der Harvardund Columbia-University im Fachblatt Science Experimente publiziert, die gezeigt haben, dass man durch Googeln weniger lernt als aus Büchern, Zeitungen oder Zeitschriften. Google taugt also vergleichsweise am wenigsten zum Erwerb von Wissen, das man schon haben muss, um überhaupt googeln zu können. Damit junge Menschen in der Schule auf die Nutzung digitaler Werkzeuge gut vorbereitet werden, dürfen wir an Schulen eines nicht tun: googeln. Und eines brauchen wir nicht tun, weil das gar nicht geht: googeln lernen. Mittlerweile gibt es eine Metaanalyse zur Frage, ob von Büchern (aus Papier) oder Bildschirmen besser gelernt werden kann. Die Antwort von mehreren Dutzend Studien an 171055 Personen ist eindeutig: von Büchern [5]. Das mag vielen Lesern zunächst paradox erscheinen, liegt aber ganz prinzipiell in der Natur der Sache, um dies hier geht, d. h. wie Menschen Sätze und Tatsachen überhaupt verstehen: Man braucht Wissen, um zu verstehen, und dieses Wissen kommt durch Bücher besser in die Köpfe als durch Bildschirme.

\section{Computer versus Gehirne}

Aus Sicht der Gehirnforschung kann man hierzu noch ergänzen: Gehirne lernen, indem sie sich mit den Dingen beschäftigen, indem sie „Informationen verarbeiten“, sagt man heute gern. Man lehnt sich dabei an die Sprache an, mit der wir beschreiben, was Computer tun: Informationen verarbeiten. Man kann durchaus so reden, sollte dabei jedoch nicht vergessen, dass Gehirne und Computer auf sehr unterschiedliche Weise funktionieren: Während in einem Computer die Verarbeitung und die Speicherung von Informationen räumlich und strukturell getrennt ablaufen, nämlich in der Central Processing Unit (CPU), einem Chip, der „rechnet“, und auf der Festplatte (dem Langzeitspeicher), gibt es diese Trennung im Gehirn nicht. Dort verarbeiten etwa 100 Milliarden Nervenzellen Informationen, indem sie sich elektrische Impulse gegenseitig zuspielen. Dabei laufen diese Impulse über Verbindungsstellen (Synapsen) der Verbindungskabel (Nervenfasern), wodurch sich die Stärke der Verbindungen ändert: Was benutzt wird, wird stärker, und was nicht benutzt wird, wird schwächer. Das Gehirn ändert sich also durch die Informationsverarbeitung (jegliche geistige Prozesse wie Wahrnehmen, Denken, Fühlen, Bewerten, Wollen etc.), die auf ihm läuft. Man spricht hier von Neuroplastizität. Durch sie hinterlassen verarbeitete Informationen Spuren im Gehirn, die man seit über 100 Jahren auch Gedächtnisspuren nennt. Den Vorgang der Entstehung dieser Spuren nennt man auch Lernen.

Die genannten Vorgänge laufen grundsätzlich immer ab, wenn gelernt wird, ganz egal, worum es sich handelt, Laufen, Sprechen, Latein oder Mathematik. Sie bilden die Grundlage jeglichen Ler- 
nens. Und je mehr Spuren schon da sind, desto besser geht die Informationsverarbeitung, eben weil im Gehirn die Speicherung und die Verarbeitung nicht getrennt sind (wie bei einem Computer), sondern in den gleichen Strukturen zugleich erfolgen. Die Gehirnforschung zeigt damit die biologischen Sachverhalte, die den Prozessen des Verstehens - d. h. der Hermeneutik - zugrunde liegen, in eindrucksvoller Weise auf. Sie kann damit die Hermeneutik nicht beweisen und braucht es auch gar nicht, denn um Verstehen geht es ja schon immer, auch wenn man das Gehirn verstehen will. Das ist weder widersprüchlich noch paradox (wie manchmal behauptet wird), denn man kann ja auch seine Augen (z. B. im Spiegel) mit seinen Augen betrachten - auch daran ist nichts widersprüchlich oder paradox!

„Bücher und Texte ganz allgemein sind doch auch Medien.“ Diese Aussage wird nicht selten behauptet, um zu unterstreichen, wie wichtig Medienkompetenz heutzutage sei. Man kann ihr durchaus uneingeschränkt zustimmen, wobei man dann aber zugeben muss: Die Wissenschaft vom Umgang mit Texten gibt es durchaus - schon sehr lange, nämlich seit es Texte gibt, also seit der Keilschrift und den Hieroglyphen. Man nennt sie Philologie. Und so wie wir keine neue Wissenschaft von Stoffen brauchen, die „Molekülkompetenz" heißt, weil es die Chemie schon lange gibt, brauchen wir keine Medienkompetenz, weil es die Philologie schon sehr lange gibt.

Halten wir fest: Medienkompetenz ist etwas, das es entweder gar nicht geben kann (weil eine „Wahrheitskompetenz im Allgemeinen" nicht existiert) oder etwas, das es schon sehr lange gibt und wofür man keinen neuen Namen braucht.

\section{Monetarisierung von Unverständnis}

Wie Tontafeln und Bücher sind Computer Werkzeuge, die man für bestimmte Aufgaben gut gebrauchen kann. Dies gilt natürlich auch für quantitative Analysen im Bereich des Textverstehens [15], also der Philologie, die uns heute von genau denjenigen Firmen (Google etc.) ermöglicht wird, zu deren Geschäftsmodell neben der Monetarisierung (wie man „zu Geld machen“ heute salonfähig nennt) unserer Aufmerksamkeit und damit Lebenszeit auch gezielte Desinformation und Täuschung gehören [16]. Betrachten wir hierzu abschließend das folgende kürzlich in der New York Times publizierte Beispiel.

Im digitalen Zeitalter wird man recht oft dazu genötigt, Einverständniserklärungen bzw. Nutzungsbedingungen zu unterschreiben, bei denen es sich im Grunde um Kaufverträge handelt: Man erhält bestimmte „Services“ und bezahlt mit persönlichen Daten. Man muss wissen, dass diese „Erklärungen“ zum „Datenschutz“ nicht etwa dazu gedacht sind, dem Nutzer irgendetwas zu erklären oder gar ihn vor irgendetwas zu schützen. Vielmehr sind diese Texte von Juristen für Juristen geschrieben, um Firmen davor zu schützen, von Nutzern verklagt zu werden. Sie enthalten sehr viel juristische Fachsprache (Jargon) und sind entsprechend unverständlich, wie eine Studie an 150 Erklärungen zum Schutz persönlicher Daten (privacy policies) zeigen konnte [13].

Knapp 150 solcher Erklärungen von sehr häufig genutzten Webseiten wie Google, Facebook, Airbnb oder der BBC wurden gelesen und die Lesedauer (in Minuten) gemessen. So braucht man zum Lesen der Yahoo-Einverständniserklärung 6 Minuten, bei der 


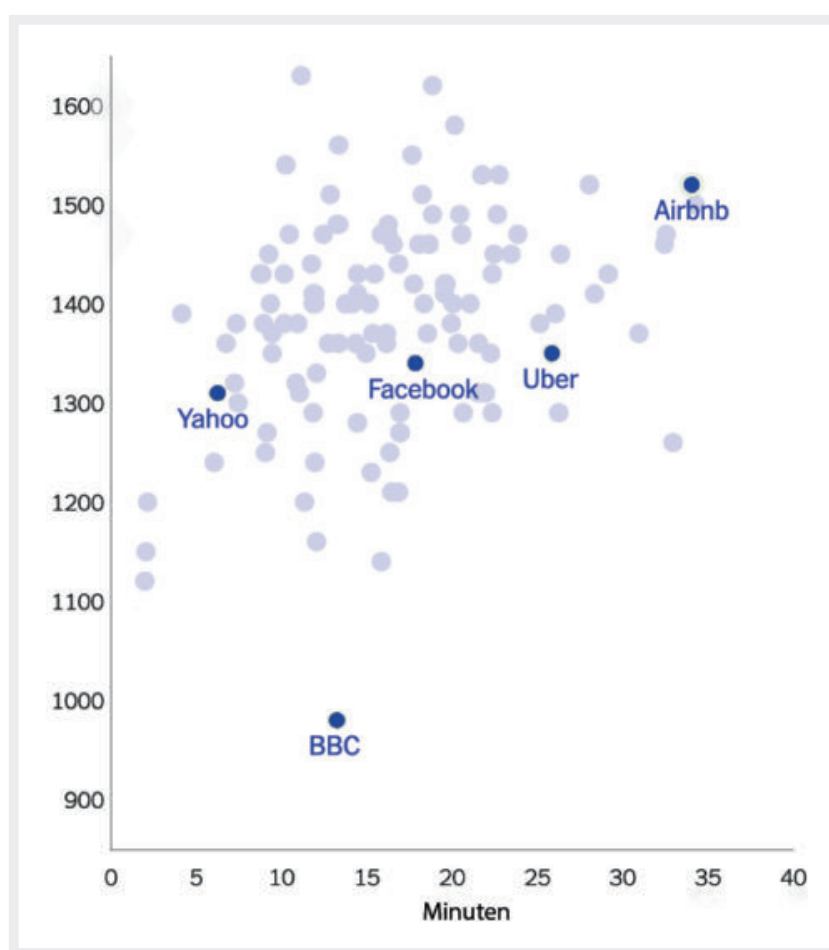

- Abb. 2 Dauer des Lesens in Minuten (X-Achse) und Schwierigkeit des Textes in Einheiten der verwendeten Software (modifiziert nach Daten aus [13]).

BBC muss man 13 Minuten lesen, bei Facebook 18 Minuten und bei Airbnb über eine halbe Stunde (was niemand macht!). Zudem wurde die Verständlichkeit (der Schwierigkeitsgrad) des Textes mithilfe der online verfügbaren Lexile Software der Bildungsfirma Metametrics untersucht. Man kopiert einfach einen Text in ein Fenster hinein, und basierend auf der Länge der Sätze und der Häufigkeit (bzw. Seltenheit) der Wörter wird automatisch ein Index der Komplexität bzw. Schwierigkeit des Textes berechnet, der von $600 \mathrm{~L}$ bis 700 L („L“ für „Lexile“) bei Drittklässlern über 850 L bis $1050 \mathrm{~L}$ bei Mittelschülern, 1050 L bis 1300 L bei Absolventen der High School, $1300 \mathrm{~L}$ bis $1450 \mathrm{~L}$ bei College-Absolventen und darüber bei Akademikern liegt ( $\triangleright$ Abb. 2).

Interessant bei der Verständlichkeit sind Vergleiche zu anderen Texten. Der Autor der Studie hat daher auch Joanne Rowlings „Harry Potter“, Stephen Hawkings „Eine kurze Geschichte der Zeit“ und Immanuel Kants „Kritik der reinen Vernunft“ - jeweils das erste Kapitel - durch die Software analysiert und fand Werte von $900 \mathrm{~L}$ für Harry Potter, 1300 L für Hawking und 1500 L für Kant. Die meisten Einverständniserklärungen setzen also mindestens einen College-Abschluss und nicht selten einen Universitätsabschluss voraus, um sie zu verstehen.

Philologen wissen schon lange, dass es leichte und schwierige Texte gibt, haben sich jedoch gescheut, hierfür Zahlen anzugeben, weil sie wissen, dass es grundsätzlich vom Leser abhängt, wie schwer oder leicht ein Text zu lesen ist. Für einen Mediziner ist ein medizinischer Text „leicht“ zu lesen, ein juristischer nicht. Und umgekehrt. Dennoch bin ich für die heutigen Methoden von mit Computern arbeitenden Philologen dankbar, da hierdurch gezeigt werden kann, dass sich Kaufverträge zuweilen etwa so schwer lesen 


\begin{tabular}{|c|c|c|c|c|c|}
\hline$\forall / 4$ & $\longrightarrow \mathrm{A}$ & 8 & -1 & H & $r$ \\
\hline $9 / 4$ & $\longrightarrow B$ & $F$ & -1 & I & Q \\
\hline$\wedge / 7$ & $\longrightarrow \mathrm{C}$ & $\omega / \psi$ & 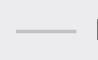 & K & 4 \\
\hline$\nabla$ & D & 6 & 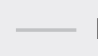 & L & $\varepsilon$ \\
\hline$E$ & $-\mathrm{E}$ & $w w / 3$ & 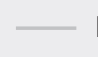 & $M$ & $t$ \\
\hline$Y$ & $\longrightarrow F$ & $4 / 2$ & 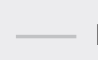 & $\mathrm{N}$ & \\
\hline$I$ & $\longrightarrow \mathrm{G}$ & $\odot / 0$ & 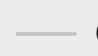 & 0 & \\
\hline
\end{tabular}

- Abb. 3 Das „Ur-Alphabet“ entstand aus Hieroglyphen und damit letztlich Bildern, die man mit etwas Fantasie in den Zeichen noch entdecken kann: Der Ochse beim A, Wasser (Wellen) beim M, das Auge beim $O$ (nach Daten aus [2]).

können wie Kants Kritik der reinen Vernunft. Hier wird, wie man das von Versicherungen, Kreditgebern und anderen Institutionen schon kennt, aufgrund von Profitstreben das Verständnis von Texten erschwert.

Dies aber bedeutet, dass diese Einverständniserklärungen nach der Europäischen Datenschutzgrundverordnung gar nicht rechtens sind. Da seit dem 1. Januar 2020 auch im US-Bundesstaat Kalifornien ein neues Gesetz zum Schutz der Privatsphäre in Kraft trat, das nach dem Vorbild der europäischen Datenschutz-Grundverordnung [16] formuliert wurde (und als das bisher strengste in den USA gilt), hat Google seine Erklärung schon vor Monaten gekürzt. Sie war vor 20 Jahren mit 2 Minuten Lesezeit (im Jahr 1999) noch einfach, mit einer halben Stunde Lesezeit im Jahr 2018 nicht mehr. Die anderen werden nachziehen müssen, um Klagen durch die EU zu vermeiden.

\section{Das Alphabet heute}

Warum verwenden wir eigentlich nicht mehr die Hieroglyphen oder die Keilschrift? Schriften entstanden nach heutiger - noch immer sehr kontrovers diskutierter Auffassung - als Abfolge von Bildern, später von Symbolen, die direkt für etwas standen (Ideo- oder Logogramme), also eine Bedeutung hatten. Schriften mit Zeichen für Sprachlaute (Phonogramme, die für Phoneme oder Silben stehen können) entwickelten sich noch später. Die Verbildlichung von Lauten, die zur Bezeichnung einer Sache oder einer Idee in einer Sprache verwendet werden, setzt eine tiefere Analyse (der Laute) der Sprache voraus - sagen die einen. Die anderen (z. B. die Chinesen) halten das Schreiben von Lauten für „oberflächlich“ (geschrieben wird ja „nur“ der Klang), das Zusammenfügen von (teilweise sehr stilisierten) grafischen Elementen zu Piktogrammen hingegen als „tief“, weil den Zeichen die Bedeutung ja direkt anzusehen ist, ohne den „Umweg“ über Laute. ${ }^{4}$

Ganz allgemein gilt, je mehr unterschiedliche Zeichen es für jeweils einzelne Bedeutungsgehalte (Logogramme) gibt, desto kür-

4 Chinesen aus den verschiedensten Provinzen, in denen die einzelnen Wörter mitunter völlig verschieden ausgesprochen werden, können sich daher verständigen, indem der Sprecher die Zeichen auf die Hand oder irgendeine Unterlage mit dem Finger „schreibt“ und der Zuhörer diese Zeichen liest. Das kann sehr schnell gehen, fast wie ein Dialog. zer wird ein Text (Beispiel: Im Chinesischen kann ein Satz aus wenigen Zeichen, die jeweils ein ganzes Wort meinen, bestehen). Schreibt man den gleichen Satz mit einem Alphabet, ist er deutlich länger. Andererseits ist man mit dem Lernen von etwa 30 Buchstaben recht schnell fertig - hierzulande nach etwa einem Schuljahr -, wohingegen die Chinesen die gesamte Grund- und Hauptschulzeit mit dem Erlernen der Schrift verbringen. Silbenschriften sind für Kinder am leichtesten zu lernen, was man daran sieht, dass in Japan die 3-Jährigen schon eine der dort gebräuchlichen Silbenschriften (Hiragana) zu lesen beginnen.

Wie also kam es zum Alphabet? Nach jüngsten Publikationen hierzu wurde es gerade nicht dort erfunden, wo man schon schreiben konnte. Welcher Hieroglyphen- oder Keilschriftspezialist, so wird dies leicht verständlich, hätte sich eine stark vereinfachte Schrift ausgedacht, die sein Expertentum obsolet machen würde? Das Alphabet wurde daher weder in Ägypten noch in Mesopotamien erfunden, sondern etwa auf halbem Weg dazwischen, wahrscheinlich - so paradox es klingen mag - von Analphabeten [2].

Im Jahr 2006 publizierte Funde dessen, woraus später das Alphabet wurde, wurden auf etwa 1800 Jahre v. Chr. datiert (Zeit der Herrschaft des Pharao Amenemhat III., vor 3800 Jahren), also etwa 1000 Jahre nach Erfindung von Hieroglyphen und Keilschrift (welche früher war, ist bis heute nicht geklärt). Es handelt sich um 2 Inschriften in Stein aus der Gegend von Luxor (dem früheren Theben), durch Militärschreiber, die einerseits eine bestimmte Form von Hieroglyphen (hieratisch) verwendete, aber andererseits aufgrund der militärischen Verwendung nicht in der Sprache der Ägypter abgefasst war. Das Heer des Pharaos muss damals eine zusammengewürfelte Mannschaft aus den verschiedensten Gebieten gewesen sein, in denen verschiedene Sprachen gesprochen wurden. So brauchte es eine Art „vereinfachter Gebrauchsschrift“ für die multilinguale Kommunikation, die wahrscheinlich auf ägyptische Schreiber einerseits und Militärs aus Gebieten von Süd-West-Asien, über Ägypten und Nubien (dem südlichen Ägypten zwischen dem 24. und 18 Breitengrad) bis zum heutigen Libyen zurückgeht. Gemäß einer konkurrierenden Theorie entstand das Alphabet auf der Sinai-Halbinsel, wo sich Minenarbeiter medial bemerkbar machen wollten, jedoch - als Mitglieder der unteren Klasse - nicht schreiben konnten. So entwickelten sie mit der Hilfe von Ägyptern, die schreiben konnten, eine einfache Schrift: das Alphabet ( Abb. 3).

Im Jahr 2015 wurde ein weiterer Fund (aus der Zeit vor 3450 Jahren) aus der Gegend um Theben publiziert, der Wörter in alphabethischer Reihenfolge (also etwa „Adele bläst crescendo damit eine Flöte gleich härter intoniert jauchzend klingt") enthält, was klar auf das Vorhandensein und den Gebrauch des Alphabets hinweist. Bereits im Jahr 2009 wurde der Befund publiziert, dass assyrische Tontafeln am Rand alphabetisch beschriftet worden waren - wahrscheinlich, weil die in der Keilschrift bewanderten Bibliothekare das „Ordnen der Buchrücken“ nach dem Alphabet leichter fanden [2]. Schließlich wurde 2018 deutlich, dass das Alphabet von vor etwa 3800 bis 3200 Jahren zwar existierte, jedoch von den Schriftgelehrten in Ägypten und Mesopotamien nicht für „amtliche“ Zwecke verwendet wurde [9]. Dies erklärt auch die geringe Standardisierung der (insgesamt nicht sehr vielen bekannten) Texte aus dieser Zeit: Man schrieb von rechts nach links oder von links nach rechts oder von oben nach unten. Dies änderte sich erst 
in den Jahrhunderten danach, und aus den Anfängen entwickelte sich das phönizische Alphabet, aus dem wiederum u. a. das griechische und damit das kyrillische und römische Alphabet hervorgingen. Es gab auch ein Keilschrift-Alphabet, das jedoch erst nach dem „Buchstaben“-Alphabet und nach dessen Muster in Mesopotamien vor weniger als 3000 Jahren entstanden war [3].

\section{Die Schulen heute}

In den Schulen wird noch heute mit der Hand geschrieben. Das gilt Vielen als anachronistisch, weil es ja mittlerweile Tastaturen und Tablets mit berührungsempfindlichen Bildschirmen gibt, die schnelleres Schreiben erlauben. In den USA hat man daher in 47 Staaten die Handschrift aus dem Pflicht-Curriculum der Grundschule gestrichen: Man kann zwar noch die Handschrift lehren und lernen, muss aber nicht. Ist das sinnvoll?

Schon lange gibt es Hinweise darauf, dass die motorische Entwicklung eines Menschen sein Denkvermögen positiv beeinflusst (Piaget). Im Bereich der Kognitionswissenschaften wurde dies durch Theorien zu „embodied cognition“ [ $[11,12]$ ebenso verdeutlicht wie durch neuere Erkenntnisse zur kindlichen Entwicklung [6, 17]. Computersimulationen des Erlernens von Buchstaben mit neuronalen Netzwerken konnten zeigen, dass bei deren rein visuellem Training die Leistungen weniger gut waren als bei Training mit zusätzlicher Komponente der motorischen Produktion von Buchstaben (mit $4 \%$ Fehler etwa so gut wie menschliche Gehirne) [10]. Dass Kinder, die mit dem Griffel schreiben lernen, Buchstaben besser erkennen und räumlich-visuelle Fähigkeiten besser entwickeln als wenn sie auf einer Tastatur tippend schreiben lernen, wurde erst kürzlich durch meine Mitarbeiter in Ulm experimentell nachgewiesen [14].

Fassen wir zusammen: Seit Jahrtausenden schreiben Menschen Texte und beschäftigen sich mit ihnen. Das nennt man Philologie. Seit einigen Jahrzehnten werden auch digitale Werkzeuge (Computer, Internet) verwendet, um Texte zu speichern, zu transportieren und zu analysieren. Die Art, wie wir Menschen Texte ganz prinzipiell verstehen, hat sich durch diese Werkzeuge nicht geändert. Gehirne machen keine Downloads, sondern werden gebraucht und ändern sich dadurch. Dieser Prozess heißt Lernen. Seit Jahrhunderten werden schwer verständliche Texte geschrieben - entweder weil die Sachen tatsächlich schwer zu verstehen sind oder um ein Informationsgefälle herzustellen, das sich monetarisieren lässt. Dagegen können und sollten wir uns wehren. Medienkompetenz kann uns dabei nicht helfen, denn es gibt sie gar nicht, es sei denn, man meint damit die Philologie. Seit 4000 Jahren lernen junge Menschen im mittleren Alter von 6 Jahren in Schulen die Schrift, was sie nicht nur zum Lesen und Schreiben befähigt, sondern auch ihre motorischen und kognitiven Leistungen (d. h. ihr Denkvermögen) verbessert. Dies tiefgreifend zu verändern und dabei zugleich dem Lernen zu schaden, ist keine gute Idee.
[1] https://hub.lexile.com/analyzer

[2] Barras C. Who invented the alphabet? The untold story of a linguistic revolution. New Scientist 5.2.2020. www.newscientist.com/article/ mg24532680-600-who-invented-the-alphabet-the-untold-story-of-alinguistic-revolution/; abgerufen am 9.2.2020

[3] Boyes PJ, Steele PM. Introduction: Issues in studying early alphabets. In: Boyes PJ, Steele PM (Hrsg.): Unserstanding Relations between Scripts II. Early Alphabets. Oxford: Oxbow Books, 2020

[4] Cancic-Kirschbaum E, Kahl J. Erste Philologien. Archäologie einer Disziplin vom Tigris bis zum Nil. Tübingen: Mohr Siebeck, 2018

[5] Delgado P, Vargas C, Ackerman R, et al. Don't throw away your printed books: A meta-analysis on the effects of reading media on reading comprehension. Educational Research Review 2018; 25: 23-38

[6] Gopnik A, Meltzoff AN, Kuhl PK. The Scientist in the Crib: What Early Learning Tells Us About the Mind. Perennial: William Morrow Paperbacks; 2000

[7] Horstmann A. Philologie. In: Ritter ], Gründer K et al. (Hrsg.): Historisches Wörterbuch der Philosophie 1989, 7: 552-572

[8] Kiefer M, Sim E-J, Liebich S, et al. Experience-dependent plasticity of conceptual representations in human sensory-motor areas. Journal of Cognitive Neuroscience 2007; 19: 525-542

[9] Koller A. The Diffusion of the Alphabet in the Second Millennium BCE: On the Movements of Scribal Ideas from Egypt to the Levant, Mesopotamia, and Yemen. Journal of Ancient Egyptian Interconnections 2018; 20(1): 1-14

[10] Lake BL, Salakhutdinov R, Tenenbaum JB. Human-level concept learning through probabilistic program induction. Science 2015; 350: 1332-1338

[11] Lakoff G. Women, Fire, and Dangerous Things: What Categories Reveal about the Mind. Chicago: University of Chicago Press, 1990

[12] Lakoff G, Johnson M. Metaphors We Live By. Chicago: University of Chicago Press, 2003

[13] Litman-Navarro K. We read 150 privacy policies. They were an incomprehensible disaster. The New York Times 2019. www.nytimes.com/ interactive/2019/06/12/opinion/facebook-google-privacy-policies. html; abgerufen am 8.2.2020

[14] Mayer C, Wallner S, Budde-Spengler N, et al. Literacy Training of Kindergarten Children With Pencil, Keyboard or Tablet Stylus: The Influence of the Writing Tool on Reading and Writing Performance at the Letter and Word Level. Front Psychol 2020; 10: 3054. doi: 10.3389/fpsyg.2019.03054

[15] Spitzer M. Aschenputtel als Flugsimulator. Nervenheilkunde 2011; 30: 545-554

[16] Spitzer M. www (WeltWeite Werbung) und die Folgen. Radikalisierung, Spionage, Vertrauens- und Wahrheitsverlust. Nervenheilkunde 2018; 37: 303-311

[17] Stahl AE, Feigenson L. Observing the unexpected enhances infants' learning and exploration. Science 2015; 348: 91-94

[18] U.S. Department of Education, National Center for Education Statistics, Program for the International Assessment of Adult Competencies (PIAAC). U. S. PIAAC 2012/2014 Results https://nces.ed.gov/surveys/ piaac/results/summary.aspx; abgerufen am 8.2.2020

[19] Waetzoldt H, Cavigneaux A. Schule. Reallexikon der Assyriologie und vorderasiatischen Archäologie 9, 294-309. Bayrische Akademie der Wissenschaften, 2009 http://publikationen.badw.de/de/rla\%7BLemmata\%7D\%5Balle\%20Rechte\%20vorbehalten\%5D.index.htm; angerufen am 29.2.2020 